\title{
1 The next steps in the study of missing individuals in networks: a comment on
}

\section{Smith et al. (2017)}

3

4

5

6

7

8

\section{Abstract}

Matthew J Silk ${ }^{1}$

${ }^{1}$ Environment and Sustainability Institute, University of Exeter, Penryn, Cornwall, UK

Corresponding author address (MJS): Environment and Sustainability Institute, University of Exeter, Penryn, Cornwall, UK matthewsilk@outlook.com

Social network analysis is now used widely to study social behaviour in humans and non-human animals, and missing individuals can represent a problem for network studies. This problem is becoming especially frequent in studies using bio-logging to collect interaction data, especially in animals. This therefore represents an important audience for Smith et al. (2017) who investigate how sub-sampling from networks impacts the outcome of subsequent analysis. Here I take advantage of the progress made by this paper to outline key issues that still require addressing to understand the effect of missing individuals on social network analysis.

Keywords: network sampling, precision, bias, accuracy, statistical modelling

\section{Introduction}

As a consequence of being relational data, the sampling of networks might inherently be expected to result in greater bias than other types of data (Alba, 1982; Silk et al., 2015; Smith et al., 2017; Smith and Moody, 2013). This could apply both to missing individuals (nodes) and missing relationships (edges), but the former is easier to quantify and address. A number of studies have explored the impact of missing individuals on network analysis, the most recent of which is Smith et 
al. (2017). The authors made substantial progress on a number of key issues, in particular in: a) assessing how non-random missingness of individuals might change the effect of sub-sampling from a network, and b) in providing a tool to allow researchers to determine the likely impact of missing individuals in a range of network structures and sizes.

Smith et al. (2017) stated that "By looking at a wide range of networks, measures and types of missing data, we can offer recommendations and best practices for applied network practitioners". A major application of network analysis away from the social sciences is in the study of animal behaviour (Croft et al., 2008; Krause et al., 2014). Missing individuals are a frequent problem in animal network studies, when it is often necessary to capture and mark individuals to gather data. However, as the use of bio-logging technology becomes more widespread to study human social networks (e.g. Isella et al., 2011; Kiti et al., 2016; Mastrandrea et al., 2015), similar problems often arise. I will provide a perspective as an applied network practitioner working on animal social behaviour as to the utility of their new findings, and then build on this to highlight important outstanding questions relating to missing individuals in networks. Finally, I will present some R code designed to test how missing individuals affect the calculation of network metrics in animal social networks that I hope will complement the java applet provided in that paper.

\section{An applied perspective on the implications of Smith et al. (2017)}

Smith et al. (2017) built upon previous work by the same authors (Smith and Moody, 2013) in exploring the consequences of missing individuals on the calculation of a range of network metrics. Together, especially when taken alongside complementary findings in other fields (e.g. (Silk et al., 2015)), the results of this work have revealed a set of important considerations when analysing networks with missing data, of which the general rules are already very useful to applied network practitioners. In particular, knowledge of how network structure and size influence the impact of sub-sampling from a network is paramount, as is an understanding that more global 
metrics (such as Betweenness) are less resilient to the presence of missing individuals. Finally, the exploration of biases in missing individuals addressed by Smith et al. (2017) is especially valuable from the perspective of animal behaviour research. This is partly as an aid in determining which individuals to collect data on when resources are limited, but also because methods of capture to make individual animals identifiable for network studies may place implicit biases on which individuals are most likely to remain unsampled. However, one opportunity that is missed here is the opportunity to discuss the importance of different types of "bias" caused by missing network data. I would like to highlight here the nomenclature/approach used by Silk et al. (2015) which looked at the effect of sub-sampling on three distinct properties of network metrics. In this paper the authors looked at precision, accuracy and bias of metric values in sub-sampled networks. As defined by these authors, precision is the correlation between values calculated in the partial (observed) network and those in the equivalent true network, accuracy is the value of the metric obtained from the observed partial network relative to its true value, and bias is systematic variation in the precision of metric values in the partial (observed) network. The impact of sub-sampling on each of these properties can depend on network structure and the type of network metric being investigated. Considering each independently is important in wider applications of network analysis, as these different properties can be used to address different questions. For example, in animal network studies it is the precision of metric values in sub-sampled networks that is important if a researcher asks a question about whether network position and a personality trait are linked, but it is accuracy that is important if a researcher seeks to compare the true values of network metrics between different contexts.

Another major step forward in Smith et al. (2017) is the development of a tool (a java applet) that can provide an idea of the impact of missing individuals (incorporating any biases in their centrality) according to network size for a range of network structures. For an applied network researcher that has worked on study systems with substantial proportions of missing individuals this is an exciting development, and has the potential to be useful as a guide to researchers designing network studies. However, from this perspective I also feel that it is essential to see a tool designed 
in this way just as a starting point. The use of network analysis in animals is now highly questiondriven and such a fixed tool has only restricted possibilities for use. It would be great to see a more modular set of functions that were able to use pilot empirical data or researcher knowledge to simulate a realistic network structure, and then sample from this structure, before determining how it might affect the outcome of a range of network analytical perspective. Such a package of functions would be best developed as a community, preferably with researchers from a range of fields so that the generation of networks, and the types of metrics to calculate (or statistical models to assess) was relevant to as wide a range of studies as possible. The advantages of taking this approach is that as a user assesses a new sampling framework and/or question, the code they used can be added to the system and shared with researchers who might be faced with a similar problem in another field.

\section{Three outstanding missing network data problems}

In this section I will highlight some important gaps in our understanding on the impact of sub-sampling from social networks that simulation-modelling could easily test and greatly aid the design of empirical studies. While these ideas come from a background of employing network analysis to study animal behaviour, I feel all are widely applicable in the use of social networks more generally.

\section{What is the best way of sub-sampling social networks?}

In many animal network studies time, cost and effort is required to capture individuals and make them individually identifiable for social network studies. This trade-off is now becoming more frequent for all types of network study, including in humans, as the use of bio-logging approaches to produce reality mining data on social behaviour is increasing (Barrat and Cattuto, 2015; Isella et al., 2011). Often these approaches are costly, and it is possible to use only relatively small sample sizes. 
Therefore, deciding how best to deploy bio-logging devices for network studies remains an open question. For example, is it best to intensively sample a small part of a network or sample a larger part of the network more sparsely? Or similarly, how would studying replicate networks in multiple populations trade-off against the intensity of collaring individuals within each population? It is likely that the type of question being asked is important to making this decision. For example, work focussing on fine-scale behavioural interactions, such as dominance behaviour in animals (e.g. Dey et al., 2015), is likely to benefit from intensively sampling particular groups. In contrast, for the study of population-level processes such as disease transmission, a more even distribution of identifiable individuals throughout a population may be beneficial, especially when attempting to record infrequent interactions.

In order to assist empirical researchers making these decisions it will be important to move simulation models of non-random sampling beyond the missingness-centrality correlation investigated by Smith et al. (2017) to assess the impacts of the clustering of identifiable and unidentifiable individuals within sub-sampled networks. Exploring this in a range of social network structures will be an important step forward in aiding the study design of network studies in natural systems, especially for studies using bio-logging approaches.

\section{How do missing individuals affect individual-level hypothesis testing in networks?}

The relationship between social network position and other individual traits has been a major cross-disciplinary research focus (e.g. Aplin et al., 2013; Bollen et al., 2011; Goodreau et al., 2009; Rosenquist et al., 2011). The most popular methods to test these hypotheses has been different, for example the use of exponential random graph models (ERGMs; Lusher et al., 2013) and stochastic actor-oriented models (SAOMs; Snijders et al., 2010) in the social sciences, versus the development of randomisation-based generalised linear mixed model approaches in animal behaviour (Croft et al., 2011; Farine and Whitehead, 2015). Regardless, the impact of missing 
individuals (and edges) on statistical inferences made using these all of these approaches remains an open and important question.

Smith et al. (2017) made a step towards addressing this, by looking at the consequences of missing individuals for tests of behavioural homophily within a network (and finding that it was possible to detect patterns of behavioural homophily when there was both a high proportion of missing individuals and a bias in which individuals were missing). However, a notion of the preponderance of type I and type II errors when different modelling frameworks are used to analyse networks with missing individuals would represent a considerable step forward in our understanding of the consequences of sub-sampling social networks. For example, while Shalizi and Rinaldo (2013) have suggested that ERGMs estimated on a sampled network are unlikely to reflect population-level hypotheses related to individual differences.

It would seem fairly simple to build on previous simulation-modelling work to examine how hypothesis testing using any of the statistical models mentioned above might be affected by the subsampling of networks. For example, the addition of a response variable that depended on network structure to the $\mathrm{R}$ function outlined in the next section would enable the impact on inference from generalised linear models to be addressed. In the case of models relating individual traits to individual-level network models, such as those suggested above, there are two main considerations to make. Firstly, the structure of the network, specifically the distribution of metrics used as an explanatory variable, is likely to influence the problems generated by sub-sampling. As a case in point, networks with higher modularity will have highly-skewed distributions for some metrics and missing individuals may reduce power considerably, especially if missingness is non-random. Secondly, the distribution of the response variable will also be an important consideration. High levels of overdispersion or zero-inflation in the response variable may exacerbate any problems associated with missing individuals. For example, individual traits with a negative binomial 
distribution (such as parasite infection load) are likely to be problematic. Again, there might be a particularly large impact on statistical power if there is a correlation between trait values and missingness.

\section{How do missing individuals affect estimates of transmission processes?}

A further major application of network analysis is to study transmission through populations; for example of information (Allen et al., 2013; Bakshy et al., 2012) and disease (Reynolds et al., 2015; Rohani et al., 2010; Stehlé et al., 2011). A case study is the application of network-based diffusion analysis (NBDA) as a powerful approach to detect social transmission in animal populations (Allen et al., 2013; Aplin et al., 2015; Franz and Nunn, 2009). Sub-sampled networks would also be expected to reduce the power of these approaches and/or lead to systematic biases in the inferences made about transmission (Ghani et al., 1998). Further type II error may result if non-random missingness means that more central individuals, which may spread information to more new individuals on average, are not identifiable. This effect of this might be particularly substantial in networks with higher modularity in which certain individuals are likely to have high brokerage between communities.

Smith et al. (2017) investigated the effect of missingness on measures of network topology that will have important implications for transmission - component size, bicomponent size and distance. All measures were highly sensitive to missing individuals (in the majority of the networks they investigated) when there was a positive missingness-centrality correlation, and even a relatively small proportion of missing nodes in this case had a substantial impact on these measures of network topology. The authors highlighted that the impact of the missingness-centrality correlation was expected in this case as more central individuals are more likely to be important to the cohesion of the network. They also suggested implications for diffusion or transmission within the network, although stopped short of using simulations to test this. 

systems prone to missing individuals such as animal populations (e.g. Hamede et al., 2012; Reynolds et al., 2015). As a result, simulation-studies that explore the impact of sub-sampling networks on conclusions drawn about transmission represent an important area of future work. It would not be too great an extension to simulate the spread of a trait across a static network and then progressively remove individuals (either at random or in a biased manner) and monitor the change in power of NBDA or similar approaches. In many cases, especially for disease transmission, the acquisition of a trait can result in potentially important changes to social network position (Ezenwa et al. 2016, Silk et al. 2017). Therefore, a further more complex extension would be to extend simulation-modelling approaches to test the impact of missing data in situations where both the network and trait were dynamic.

\section{A tool for generating and sampling from varying network structures}

In the supplementary information I present R functions for generating and sub-sampling from weighted, undirected social networks in a range of structures. Similarly to java applet provided by Smith et al. (2017) this is intended to encourage researchers to consider the potential impacts of missing nodes on their analyses (and in many situations this tool will be sufficient). However, unlike the java applet the code enables readers to generate user-defined networks and sample from them. An added advantage is that the code is designed in such a way that researchers familiar with R could add their own functions to address sampling-related problems of interest to them.

The code draws tie strengths from a zero-inflated negative binomial distribution. In its most basic form, this negative binomial distribution is equivalent for all individuals. However, I present it with two possible extensions, which will be of general interest to many network researchers. First, the tool includes code that enables generated networks to be "spatially structured" by placing individuals in $2 \mathrm{~d}$ space and basing the parameters of the negative binomial distribution on the 
distance matrix of their locations. Many social network studies take place in populations where social interactions are structured by space use, and in these contexts being able to develop simulated networks that account for this is important. Second the tool includes the ability to assign individuals into social groups of fixed or varying sizes. Different distributions of tie strengths can be set for within-group and out-of-group ties in the network allowing the generation of networks with the strong social group structure frequently found in many human and animal populations. It also provides a highly tractable way to test network sub-sampling questions in networks differing in modularity. The capability of this tool to produce a whole range of potentially realistic network structures is demonstrated in Figure 1. If preferred then networks could alternatively be generated by using an exponential random graph model to create networks with a set of desired target statistics, and the networks generated using this method could be used in the subsequent $R$ functions in the same manner.

The supplementary information also includes code that is able to sub-sample from these networks, as a simple illustration of how it can be used to address some of the issues discussed in this paper. This calculates and outputs the precision, accuracy and bias of four centrality metrics (degree, strength, betweenness and eigenvector centrality) in partial networks of pre-defined size (i.e. the proportion of the population made identifiable). The precision, accuracy and bias of these metrics calculated in the networks depicted in Fig. 1 is provided in the supplementary material. In this example networks of 50 nodes were generated using the three models specified in the legend of was then used to randomly sub-sample these networks to contain $60 \%$ and $80 \%$ of the original nodes once (it could be applied multiple times to generate repeat sampling events and facilitate 
statistical comparisons). Finally, the third R function calculated the precision, accuracy and bias (as defined above) of metric values in the sub-sampled networks (Table S1).

Currently the code is limited to random sub-sampling of nodes, and to tests of the precision, bias and accuracy of centrality metrics. However, it provides a valuable basis for developing simulation models to address some of the outstanding issues highlighted above. For example, the ability to have both spatial and modular distribution of the nodes in the network facilitates adjustments to the sampling regime that would easily enable the impact of clustered versus random sampling at different scales to be tested. Similarly, the replacement of the final R function (which currently calculates the precision, accuracy and bias of sampled networks) with an alternative function that fits statistical models (e.g. ERGMs) and compares parameter estimates at different levels of sampling would make it easily possible to determine how sub-sampling nodes affects statistical inference and hypothesis testing.

Together these R functions provide a tool to estimate the impact of sub-sampling networks in a whole-range of user-defined biologically realistic network structures. By providing modular code in GNU software I hope to provide a toll that other researchers can use in a system-specific manner according to their needs, thus complementing the java applet provided by Smith et al. (2017) which is predominantly targeted as an easy-to-use and quick guide applicable to general types of network structure. Together, with the developments in that paper, I anticipate that these R functions will trigger further work towards addressing some of the outstanding questions about sub-sampling networks highlighted previously.

\section{Conclusions}

The publication of Smith et al. (2017) advances our understanding of the impact of missing individuals on network analysis, and offers great potential to applied network practitioners outside 

of the social sciences. However, I advocate the use of approaches that move beyond calculating biases in descriptive network metrics. In particular, a move towards determining how sub-sampling

251 networks in empirical systems affects our power to use statistical models of network structure and

252 individual behaviour will be especially beneficial. This will be facilitated by more modular and

253 adaptable approaches to examining the effects of missingness in network data that can be shared

254 among researchers and will be applicable to networks in a range of fields.

255

256 
a)

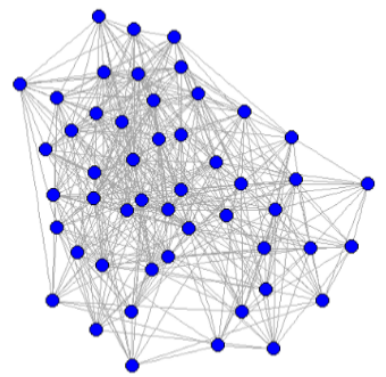

b)

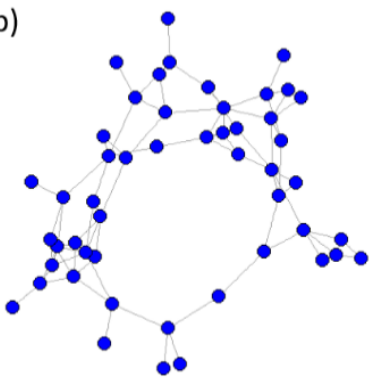

c)

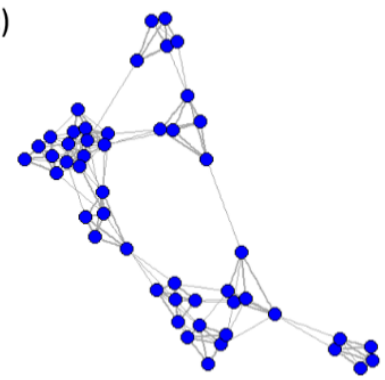

259 Figure 1. Three networks generated using the network.generator() R function provided in the 260 supplementary material. For a) groups=50, mean.group.size=max.group. $\operatorname{size}=1$, d.eff $=2$, o.dens $=0.7$ 261 and i.dens=6 (not that this parameter is used in this case). For b) groups=50, 262 mean.group.size=max.group.size $=1$, d.eff $=10$, o.dens $=0.7$ and i.dens=6 (not that this parameter is 263 used in this case). For c) groups=10, mean.group.size=max.group.size=5, d.eff=10, o.dens=1 and 264 i.dens $=6$. 
Alba, R.D., 1982. Taking stock of network analysis: A decade's results. Res. Sociol. Organ. 1, 39-74.

Allen, J., Weinrich, M., Hoppitt, W., Rendell, L., 2013. Network-based diffusion analysis reveals cultural transmission of lobtail feeding in humpback whales. Science (80-. ). 340, 485-488.

Aplin, L.M., Farine, D.R., Morand-Ferron, J., Cockburn, A., Thornton, A., Sheldon, B.C., 2015. Experimentally induced innovations lead to persistent culture via conformity in wild birds. Nature 518, 538-541.

Aplin, L.M., Farine, D.R., Morand-Ferron, J., Cole, E.F., Cockburn, A., Sheldon, B.C., 2013. Individual personalities predict social behaviour in wild networks of great tits (Parus major). Ecol. Lett. 16, $1365-1372$.

Bakshy, E., Rosenn, I., Marlow, C., Adamic, L., 2012. The role of social networks in information diffusion, in: Proceedings of the 21st International Conference on World Wide Web. ACM, pp. 519-528.

Barrat, A., Cattuto, C., 2015. Face-to-face interactions, in: Social Phenomena. Springer, pp. 37-57.

Bollen, J., Gonçalves, B., Ruan, G., Mao, H., 2011. Happiness is assortative in online social networks. Artif. Life 17, 237-251.

Croft, D.P., James, R., Krause, J., 2008. Exploring animal social networks. Princeton University Press.

Croft, D.P., Madden, J.R., Franks, D.W., James, R., 2011. Hypothesis testing in animal social networks. Trends Ecol. Evol. 26, 502-507.

Dey, C.J., Tan, Q.Y.J., O'Connor, C.M., Reddon, A.R., Caldwell, J.R., Balshine, S., 2015. Dominance network structure across reproductive contexts in the cooperatively breeding cichlid fish Neolamprologus pulcher. Curr. Zool. 61, 45-54.

Ezenwa, V.O., Archie, E.A., Craft, M.E., Hawley, D.M., Martin, L.B., Moore, J. \& White, L. (2016) Host behaviour-parasite feedback: an essential link between animal behaviour and disease ecology. Proc. R. Soc. B, p. 20153078. The Royal Society.Farine, D.R. (2013). Animal social network inference and permutations for ecologists in R using asnipe. Methods in Ecology and Evolution, 4, 1187-1194.

Farine, D.R., Whitehead, H., 2015. Constructing, conducting and interpreting animal social network analysis. J. Anim. Ecol. 84, 1144-1163.

Franz, M., Nunn, C.L., 2009. Network-based diffusion analysis: a new method for detecting social learning. Proc. R. Soc. London B Biol. Sci. 276, 1829-1836.

Ghani, A.C., Donnelly, C.A., Garnett, G.P., 1998. Sampling biases and missing data in explorations of sexual partner networks for the spread of sexually transmitted diseases. Stat. Med. 17, 20792097.

Goodreau, S.M., Kitts, J.A., Morris, M., 2009. Birds of a feather, or friend of a friend? Using exponential random graph models to investigate adolescent social networks. Demography 46 , 103-125.

Hamede, R., Bashford, J., Jones, M., McCallum, H., 2012. Simulating devil facial tumour disease outbreaks across empirically derived contact networks. J. Appl. Ecol. 49, 447-456.

Isella, L., Stehlé, J., Barrat, A., Cattuto, C., Pinton, J.-F., Van den Broeck, W., 2011. What's in a crowd? 
Kiti, M.C., Tizzoni, M., Kinyanjui, T.M., Koech, D.C., Munywoki, P.K., Meriac, M., Cappa, L., Panisson, A., Barrat, A., Cattuto, C., 2016. Quantifying social contacts in a household setting of rural Kenya using wearable proximity sensors. EPJ Data Sci. 5, 21.

Krause, J., James, R., Franks, D.W., Croft, D.P., 2014. Animal social networks. Oxford University Press.

Lopes, P.C., Block, P., König, B., 2016. Infection-induced behavioural changes reduce connectivity and the potential for disease spread in wild mice contact networks. Sci. Rep. 6.

Lusher, D., Koskinen, J., Robins, G., Lusher, D., Koskinen, J., Robins, G., 2013. Exponential random graph models for social networks. Structural analysis in the social sciences.

Mastrandrea, R., Fournet, J., Barrat, A., 2015. Contact patterns in a high school: a comparison between data collected using wearable sensors, contact diaries and friendship surveys. PLoS One 10, e0136497.

Reynolds, J.J.H., Hirsch, B.T., Gehrt, S.D., Craft, M.E., 2015. Raccoon contact networks predict seasonal susceptibility to rabies outbreaks and limitations of vaccination. J. Anim. Ecol. 84, 1720-1731.

Rohani, P., Zhong, X., King, A.A., 2010. Contact network structure explains the changing epidemiology of pertussis. Science (80-. ). 330, 982-985.

Rosenquist, J.N., Fowler, J.H., Christakis, N.A., 2011. Social network determinants of depression. Mol. Psychiatry 16, 273-281.

Shalizi, C.R. \& Rinaldo A. (2013). Consistency under sampling of exponential random graph models. Annals of Statistics, 41, 508-535.

Silk, M.J., Jackson, A.L., Croft, D.P., Colhoun, K., Bearhop, S., 2015. The consequences of unidentifiable individuals for the analysis of an animal social network. Anim. Behav. 104, 1-11. doi:10.1016/j.anbehav.2015.03.005

Silk, M.J., Croft, D.P., Delahay R.J., Hodgson, D.J., Boots M., Weber N. and McDonald R.A. (2017). Using Social Network Measures in Wildlife Disease Ecology, Epidemiology, and Management. BioScience doi: 10.1093/biosci/biw175

Smith, J.A., Moody, J., 2013. Structural effects of network sampling coverage I: Nodes missing at random. Soc. Networks 35, 652-668.

Smith, J.A., Moody, J., Morgan, J.H., 2017. Network sampling coverage II: The effect of non-random missing data on network measurement. Soc. Networks 48, 78-99.

Snijders, T.A.B., Van de Bunt, G.G., Steglich, C.E.G., 2010. Introduction to stochastic actor-based models for network dynamics. Soc. Networks 32, 44-60.

Stehlé, J., Voirin, N., Barrat, A., Cattuto, C., Colizza, V., Isella, L., Régis, C., Pinton, J.-F., Khanafer, N., Van den Broeck, W., 2011. Simulation of an SEIR infectious disease model on the dynamic contact network of conference attendees. BMC Med. 9, 1. 